\title{
A standard picture of healthy oral mucosae by direct oral microscopy
}

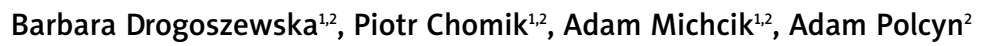 \\ 1Department of Oral and Maxillofacial Surgery, Medical University of Gdansk, Poland \\ Head: Prof. Adam Włodarkiewicz MD, DMD, PhD \\ 2Department of Otolaryngology, Ward of Maxillofacial Surgery, University Clinical Center, Gdansk, Poland \\ Head: Prof. Adam Włodarkiewicz MD, DMD, PhD
}

Postep Derm Alergol 2013; XXX, 3: 159-164 DOI: $10.5114 /$ pdia.2013.35617

\begin{abstract}
Introduction: Direct oral microscopy constitutes a novel technique of in vivo oral mucosae examination. The basic principles of this method derive from colposcopy and dermoscopy. The main goal of direct oral microscopy is the earliest possible detection of oral precancerous lesions in order to implement their treatment as quickly as possible and prevent malignant transformation.

Aim: To establish a standard picture of healthy oral mucosae with direct oral microscopy applying standard colposcopic criteria in order to create a reference point for further diagnosis of precancerous lesions.

Material and methods: Thirty patients of both genders with clinically unaltered oral mucosae were examined. For every individual, clinical examination with the naked eye was performed, followed by direct oral microscopy with colposcopic assessment criteria. Oral mucosae at various sites (lip, cheek, floor of mouth, ventral and lateral sides of the tongue, alveolar ridge and soft palate) were examined.

Results: Subepithelial blood vessel patterns, mucosal surface, colour tone and transparency were described for healthy oral mucosae. Moreover, cases with clinically unaltered oral mucosae where direct oral microscopy revealed subclinical alterations were described.

Conclusions: Direct oral microscopy with colposcopic assessment criteria enables establishment of a repeated picture of unaltered oral mucosae. The standard picture of healthy oral mucosae is an essential reference point for application of this technique to early diagnose potentially malignant oral mucosal lesions as well as apply their early treatment.
\end{abstract}

Key words: direct microscopy, oral cavity, colposcopy, dermoscopy, diagnosis.

\section{Introduction}

Direct oral microscopy constitutes a novel, yet sporadically utilised, non-invasive diagnostic technique, which aids clinical examination of the oral cavity. The basic principles of this method derive from colposcopy, a common diagnostic tool in gynaecology, and dermoscopy which is utilised in clinical evaluations of skin lesions. The oral mucosa is examined microscopically at multiple magnifications so that features such as subepithelial mucosal vessels, surface patterns, colour tone, transparency as well as the exact demarcation boundaries of mucosal lesions can be estimated. The examination of subepithelial mucosal vessels is facilitated by the application of a green filter to the light source which enhances the contrast between these vessels and the surrounding tissues [1].
The histologies of the oral mucosa and lower genital tract mucosa are similar, which led to the adaptation of noninvasive gynaecologic diagnostic tools for direct oral microscopy. Basically, there are three types of mucous membrane overlaying the oral cavity. The masticatory mucosa, whose external layer is composed of the squamous epithelium, covers sites exposed to considerable frictional forces during chewing. This kind of mucosa is localised mainly on the hard palate, the dorsal side of the tongue and around teeth [2]. Sites not directly involved in the mastication process are covered by squamous non-keratinizing epithelium [2]. The sensory oral epithelium plays an important role in the gustatory and masticatory function, overlaying mainly the dorsal surface of the tongue [2]. Keratinocytes are the basic cells constituting the oral squa-

Address for correspondence: Piotr Chomik DMD, PhD, Ward of Maxillofacial Surgery, University Clinical Center, 17 Smoluchowskiego St, 80-214 Gdansk, Poland, phone: +48 5834930 90, fax: +48 5834931 00, e-mail: piotr.chomik@gumed.edu.pl,

wlodarkiewicz@gumed.edu.pl

Received: 22.04.2013, accepted: 25.04.2013. 
mous epithelium. These cells contain keratin - a protein particularly resistant to mechanical and chemical insults, which ensures protection of oral mucosa against injuries $[2,3]$.

The basal membrane delineates the oral squamous epithelium from the proper layer of the oral mucosa (lamin a propria). It is possible to differentiate, from this layer up to the surface, specific layers of non-keratinizing epithelium: the basal layer (stratum basale), the spinous layer (stratum spinosum), the granular layer (stratum granulosum) and the desquamation layer (stratum disjunctum) $[2,3]$. In the keratinizing epithelium, the clear layer (stratum lucidum) overlays the granular layer and the corneous layer (stratum corneum) is the most superficial. The proper layer of the mucosa is composed of connective tissue rich in collagen fibres and fibroblasts, as well as macrophages, mast cells, lymphocytes and granulocytes. Networks of blood and lymphatic vessels, as well as nerves are also present. The blood vessels of the proper layer of oral mucosa, particularly their physiological and pathological patterns, are the elements observed easily by direct oral microscopy. Minor salivary glands, responsible for moisture of the mucosal surface are also present in the proper layer of the oral mucosa [2-4]. The oral mucosa performs multiple functions: protection, self-renewal, taste perception, absorption, sensation and secretion.

\section{Aim}

A study of the available literature revealed few reports describing the use of direct microscopy, termed colposcopy, to diagnose potentially malignant disorders of the oral mucosa [1, 5-7]. A pilot study was published by Gynther et al. in 2000 [1]. The aim of our study was to establish a standard picture of healthy oral mucosae by direct microscopy, applying standard colposcopic criteria as used in the above study. Furthermore, we subjected abnormalities found in our in vivo microscopic pictures of the oral mucosa (which were not visible during examination with the naked eye) to colposcopic criteria. The potential of these abnormalities to trigger malignant transformations is discussed.

\section{Material and methods}

Adult patients, aged 37-61 years (mean age 49 years) referred to the Outpatient Clinic of Maxillofacial Surgery and Ward of Maxillofacial Surgery at the Department of Otolaryngology, University Clinical Centre in Gdansk, Poland, were included in the study. A total of 30 patients (16 males, 14 females) presenting clinically unaltered oral mucosae, treated due to maxillofacial trauma and/or cystic tumours of maxillofacial skeleton were enrolled. Information concerning general health, smoking habits, alcohol consumption and other addictions was collected for every individual. Before the study started, every patient gave written consent to the examination having been informed about the procedure and aim of the study. The study design was accepted by the Institutional Review Board of Medical University in Gdańsk (NKEBN/137/2011).

Patients with macroscopically unaltered oral mucosae were thoroughly examined under a halogen lamp with the naked eye to confirm the absence of mucosal disorders. Eventually, direct oral microscopy was performed in a darkened room to establish a standard picture of healthy oral mucosae according to colposcopic criteria (subepithelial mucosal vessels, surface pattern, colour tone, transparency). Patients who presented deviations from the standard mucosal picture observed by direct oral microscopy were biopsied under local anaesthesia with $2 \%$ lignocaine.

Direct oral microscopy was performed with a Leisegang colposcope, model BG/LED Y/C type 3ML (Leisegang, Germany). Photographs were taken with a Canon EOS 500D digital camera equipped with a Canon Macro $100 \mathrm{~mm}$ lens (Canon, Japan). An Apple iMac computer with Mac OS X software (Apple, CA, USA) was utilised for analysis, processing and archiving of pictures.

\section{Results}

Analysis of direct microscopy pictures at magnifications of $7.5 \times, 15 \times$ and $30 \times$ in 30 patients presenting with clinically unaltered oral mucosae and not complaining about mucosal disorders was performed. Every colposcopic checkpoint mentioned was described for each individual: subepithelial blood vessel pattern and density, surface texture, colour and transparency. Oral mucosa at various sites (lip, cheek, floor of mouth, ventral and lateral sides of the tongue, alveolar ridge and soft palate) were examined.

Subepithelial blood vessels in healthy oral mucosae, distinct using the green filter, showed patterns of network capillaries or hairpin capillaries. Network capillaries were seen in 11 patients (36.7\%), whereas hairpin capillaries were observed in 19 patients (63.3\%). Such patterns remain in accordance with the colposcopic criteria for healthy uterine cervix mucosae, without any suspicion of dysplasia. Subepithelial blood vessels were especially visible in the buccal mucosa. In patients with a thin mucosal biotype, these blood vessels appeared through the epithelium and were visible without use of the green filter. Application of the green filter, however, gave better contrast between capillaries and the surrounding tissue, which resulted in more accurate pattern descriptions (Figures 1 and 2). In uterine cervix colposcopy, if subepithelial blood vessels are observed without using the green filter, it may indicate the presence of an atrophic squamous epithelium. This condition is still classified colposcopically correct however, unless co-existing deviations are visible.

Healthy oral mucosae presented moist, glossy, smooth and non-folded surfaces using direct oral microscopy. At particular sites individual foci of epithelial disjunction were 


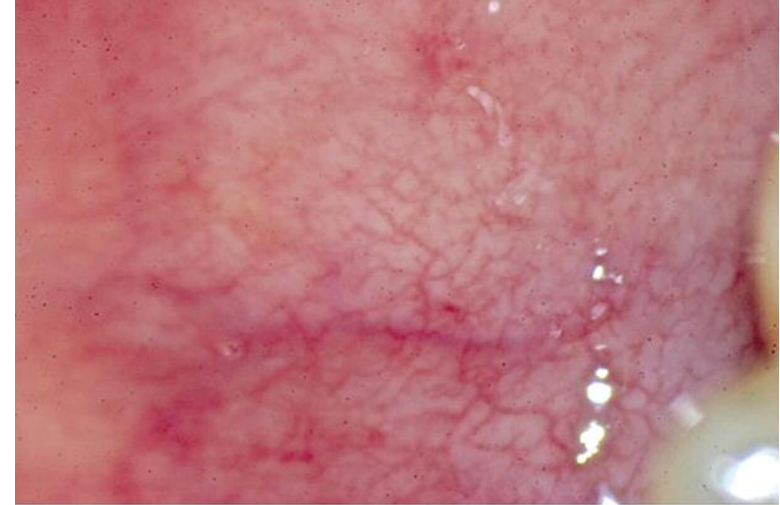

Fig. 1. Subepithelial capillaries in buccal mucosa of thin biotype are visible without the application of green filter (magnification 30x)

observed which expresses self-renewal abilities of oral squamous epithelium (Figure 3). This feature was seen in 13 patients (43.3\%), superficial epithelial disjunctions were not observed in the remaining 17 individuals (56.7\%). In colposcopic examination of uterine cervix, self-renewal abilities of squamous epithelium are expressed by the presence of subtle foci of whitening within immature metaplastic epithelium comparable to foci of oral epithelial disjunction mentioned above. This fulfils the criteria of a correct colposcopic picture of uterine cervix.

Healthy oral mucosae presented a range of colours from pale-rosy to rosy by direct oral microscopy, contrasting sharply with red subepithelial capillaries, which were particularly visible after application of the green filter (Figures 4 and 5). Such colouration remains in accordance with the colposcopic criteria of healthy cervical mucosa with single foci of whitening, particularly visible after the acetic test, being a sign of progressive regeneration within immature metaplastic epithelium. A pale-rosy colouration of the oral mucosa was observed in 8 individuals (26.7\%) and a rosy colour detected in the remaining 22 patients (73.3\%).

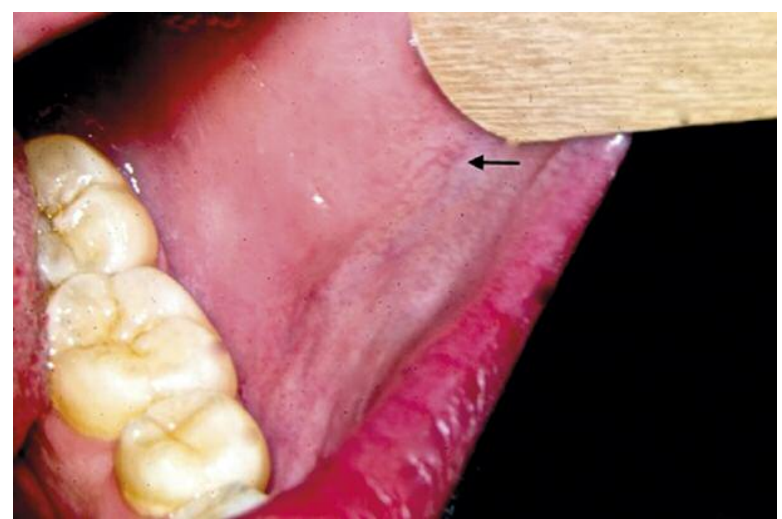

Fig. 4. Pale-rosy colouration of healthy buccal mucosa. The arrow indicates subepithelial capillaries in proximity to oral angle (magnification 7.5×)

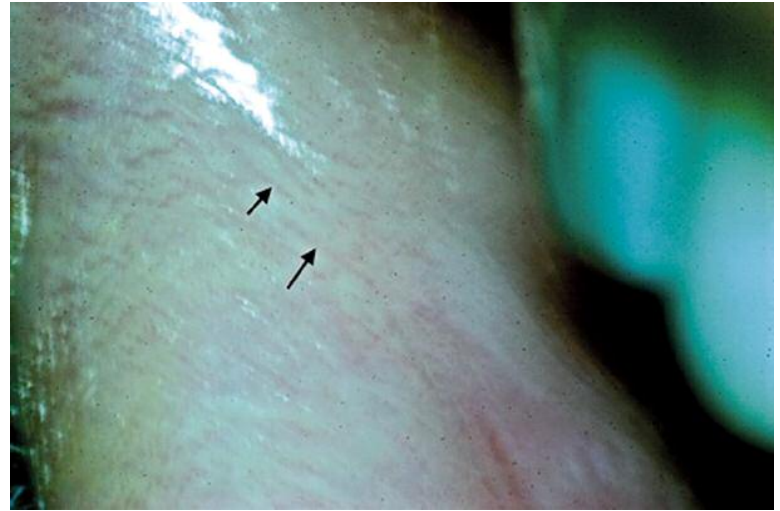

Fig. 2. Single, thin subepithelial capillaries of lower lip mucosa (arrows) visible in green filter. Hairpin pattern of capillaries (magnification 30x)

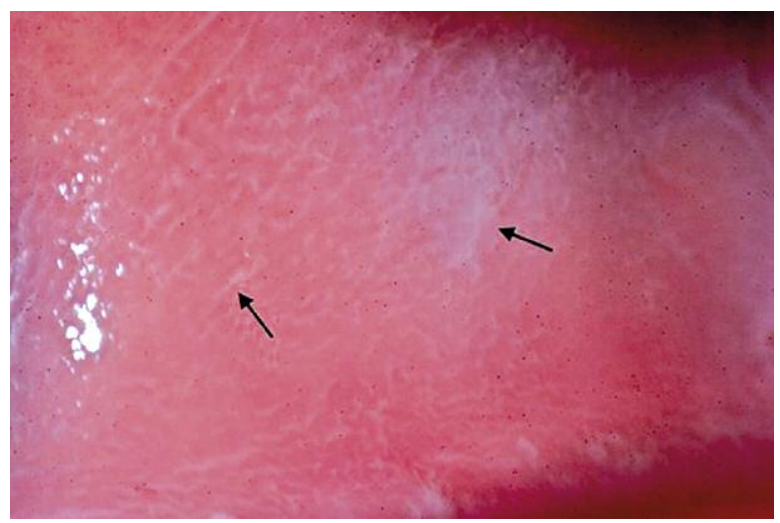

Fig. 3. Foci of disjunction in superficial epithelial layers of buccal mucosa (arrows) express epithelial self-renewal abilities (magnification 30x)

A thin biotype and partial to total transparency were detectable by direct oral microscopy in healthy oral mucosae. In total transparency, which was observed in 10 cases (33.3\%), subepithelial capillaries were visible without use

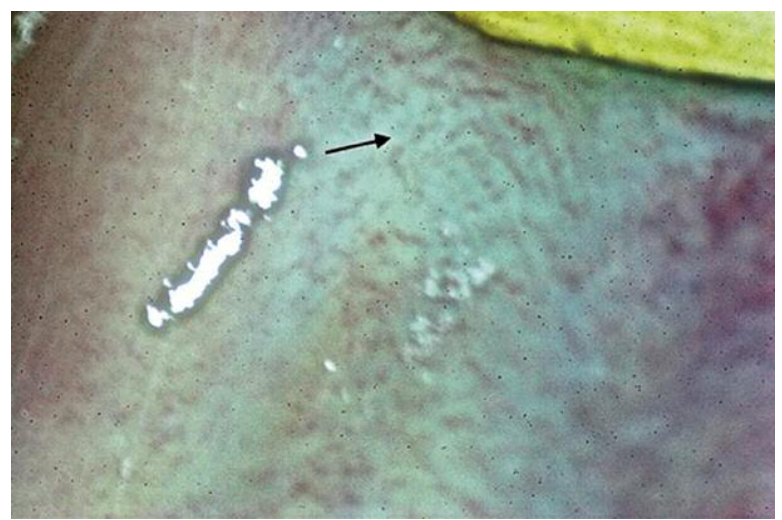

Fig. 5. The application of green filter increases the contrast between red subepithelial capillaries (arrow) and rosy oral mucosa (magnification 30x) 


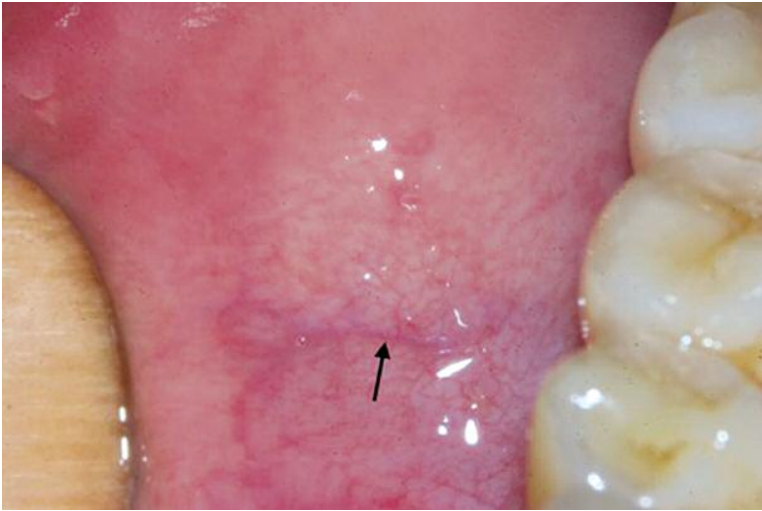

Fig. 6. Total transparency of healthy oral mucosa enables observation of subepithelial capillaries without the application of green filter. Subepithelial capillaries are visible against a background of larger vessel (arrow) in submucosa (magnification 15x)

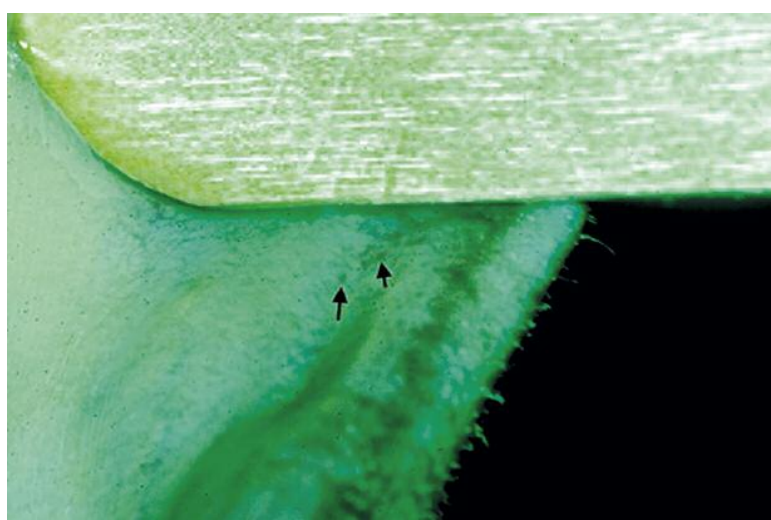

Fig. 8. Subepithelial single punctation capillaries (arrows) in buccal mucosa visible in green filter in the patient with clinically unaltered oral mucosae (magnification 30x)

of the green filter. Partial transparency of the oral mucosa was defined as a state when subepithelial vessels were not visible with the white light of the microscope, however application of the green filter enabled their visualisation, which fulfils the colposcopic criteria of healthy mucosae (Figures 6 and 7). Partial transparency of healthy oral mucosae was observed in 20 individuals (66.7\%). In colposcopic examination of uterine cervix, visibility of subepithelial capillaries without the green filter expresses lightening of squamous epithelium, which is particularly typical of the postmenopausal period and does not indicate pathology.

Direct oral microscopy also revealed lesions, subclinical deviations according to colposcopic criteria, which were not observed macroscopically. These were mainly subepithelial punctation and mosaic capillaries. Lesions were identified in 9 patients altogether, 7 of whom (77.8\%) presented subepithelial punctation capillaries (Figure 8 ) and the remaining 2 individuals (22.2\%) revealed mosaic capillaries (Figure 9). Biopsies obtained from these 9 patients revealed no signs of dysplasia in 3 cases (33.3\%), which included 2 people

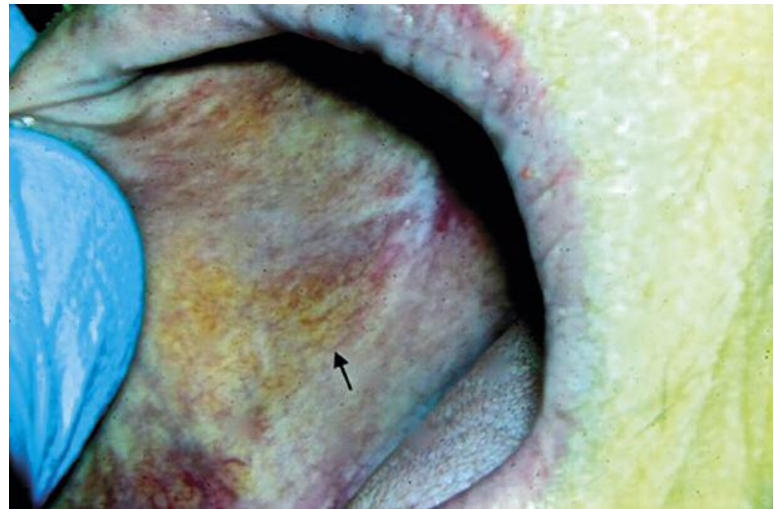

Fig. 7. Improved visibility of subepithelial capillaries (arrow) due to increased contrast between the vessels and surrounding mucosa (magnification 15×)

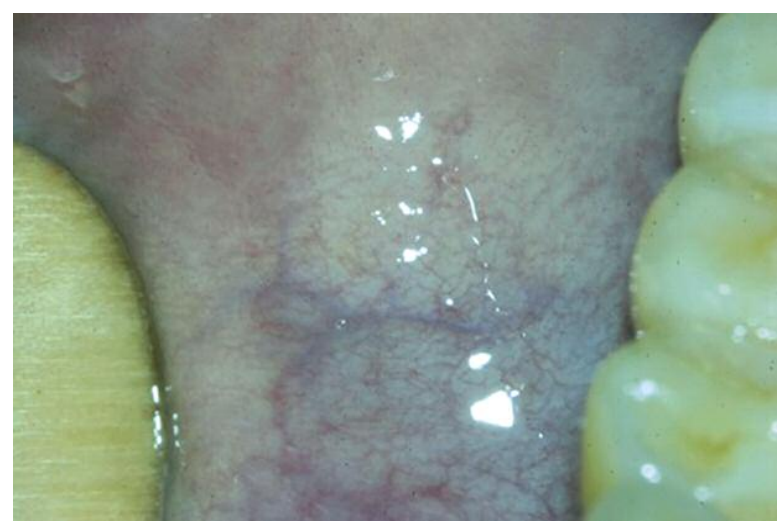

Fig. 9. Subepithelial mosaic capillaries in the patient with clinically unaltered oral mucosae visible in green filter (magnification 15x)

(22.2\%) with punctation capillaries and 1 person (11.1\%) with mosaic capillaries. In the remaining 6 cases (66.7\%), signs of dysplasia were detected. Mild dysplasia in 5 patients (55.6\%) who presented punctation capillaries and moderate dysplasia in 1 patient (11.1\%) who presented mosaic capillaries (Table 1). For these 9 patients, the other parameters of mucosal estimation by direct oral microscopy were not different from the established standard of healthy mucosae.

\section{Discussion}

In vivo imaging techniques are increasingly popular in modern medicine. Constant progress in biomedical engineering technologies generates more accurate results, which in turn improves clinical evaluation of patients and histopathological specimen interpretation. So far, these methods have been successfully applied in specialities such as gynaecology and dermatology. Colposcopic examination together with dye tests has been routinely performed to improve clinical gynaecological evaluation. It has been re- 
cognised as crucial in cases of incorrect or unsatisfactory cytology of the uterine cervix [8]. The long-term application of colposcopy in a wide range of gynaecologic diagnostic tools as well as the availability of more and more sophisticated equipment has contributed to a relatively high sensitivity and specificity of this diagnostic method [9].

Dermatoscopy (epiluminescence microscopy - ELM) has achieved similar significance in dermatology. Particular effectiveness of this method was demonstrated in the diagnosis of melanocytic lesions, skin cancers and melanomas, as well as in clinical differentiation between benign skin lesions and those suspected of malignant transformation [10]. The application of dermatoscopy to diagnose oral mucosal melanocytic lesions has been reported [11-14]. This method was shown to be particularly effective in the estimation of the borders of pathologic lesions [15].

Technological progress in recent years has resulted in the development of sophisticated tools dedicated to in vivo diagnostic procedures of the oral cavity at high magnifications. Reports describing in vivo imaging of the oral mucosa in search of potentially malignant lesions are still rare in literature, though. In this work, based upon colposcopic criteria and the pilot study of Gynther et al. [1], we observed healthy oral mucosae by direct oral microscopy. We were able to visualise subepithelial blood vessels, especially when a green filter was applied, detecting subepithelial network capillaries and hairpin capillaries in healthy oral mucosa. In the vaginal portion of the uterine cervix, subepithelial vessels are not visible in white light, whereas with a green filter, network or shrubby capillaries are revealed, which are similar to those observed in the oral cavity [16, 17]. The visibility of normal subepithelial blood vessels by colposcopic examination without the green filter indicates lightening of cervical epithelium as a sign of its spontaneous regeneration during the menstrual cycle or after surgical procedures [18]. It may also indicate epithelial atrophy typical of the postmenopausal period and physiologically those vessels may become visible in the second phase of the menstrual cycle or in pregnancy [17]. The common feature of the gynaecological conditions described above is the lack of any signs of oncogenesis associated with network or hairpin capillaries. This is in agreement with the observations of this study, where network or hairpin capillaries were observed only in cases of healthy oral mucosa.

Healthy oral mucosae presented smooth, non-folded, moist and glossy surfaces after observation by direct oral microscopy. Sites exposed to mechanical contact with teeth and food on the buccal mucosa in some patients presented discrete foci of disjunction and a whitening of the superficial layers of the oral epithelium (stratum disjunctum). These regions represent the regeneration of the superficial layers of the oral epithelium, demonstrating its protective function on oral cavity tissues [2, 3]. Comparing these images to colposcopic criteria, we can assume that these regions are typical of the so-called "regeneration zone". This is the site of cervical epithelial transformation,
Table 1. Combination of features observed in direct oral microscopy in cases of clinically unaltered oral mucosae

\begin{tabular}{lccc}
\hline $\begin{array}{l}\text { Grade } \\
\text { of dysplasia }\end{array}$ & $\begin{array}{c}\text { Punctation } \\
\text { capillaries }\end{array}$ & $\begin{array}{c}\text { Mosaic } \\
\text { capillaries }\end{array}$ & Total \\
\hline No dysplasia & $2 / 9(22.2 \%)$ & $1 / 9(11.1 \%)$ & $3 / 9(33.3 \%)$ \\
\hline Low-grade dysplasia & $5 / 9(55.6 \%)$ & - & $5 / 9(55.6 \%)$ \\
\hline Moderate dysplasia & - & $1 / 9(11.1 \%)$ & $1 / 9(11.1 \%)$ \\
\hline & & & $9 / 9(100 \%)$ \\
\hline
\end{tabular}

where the cylindrical epithelium of the uterine cervix canal is gradually replaced by secondary squamous epithelium within the vaginal portion of uterine cervix [19]. An immature metaplastic squamous epithelium is not considered as precancerous tissue in such instances, but, similarly to the oral cavity, indicates the regenerative abilities of this anatomical site [17]. Beyond the regeneration zone, the healthy mucosa of the vaginal portion of the uterine cervix is smooth, non-folded, glossy and moist, similar to the healthy mucosa of the oral cavity [16].

The colour of oral mucosae in direct microscopy was described as pale-rosy to rosy, in accordance with colposcopic criteria, where a healthy epithelium is described as rosy to pale red [20]. Whiter areas within the healthy epithelium of the vaginal portion of the uterine cervix are typical of the regeneration zone mentioned above or atrophic epithelium, whereas red colouration within the epithelium heralds inflammation, not necessarily associated with oncogenesis. The red colour results from terminal capillaries being overloaded with blood, which is seen through the squamous epithelium [17]. An intensive red colour may also indicate ectopy, which is the displacement of glandular epithelial cells from the uterine cervix canal to its vaginal portion [21]. Such a condition is considered as paraphysiological and not pathological. In this study the presence of an intensive red oral mucosa without coexisting clinical and subclinical features of precancerous lesions suggested the presence of oral cavity inflammation mostly of odontogenic origin.

The transparency of the oral mucosa was defined as total, when subepithelial vessels were visible without the green filter in direct oral microscopy. Partial transparency was defined as the situation, when subepithelial structures became visible after application of the green filter. Oral mucosae, which presented partial to total transparency in direct oral microscopy were considered healthy. Total transparency was associated with a thin biotype of oral mucosae, when all layers, including the epithelium, presented a decreased thickness [22]. A thin biotype was not considered as pathological, but an individual feature. Comparing our images to gynaecologic and colposcopic criteria it is assumed that healthy cervical mucosae present mostly partial transparency, which means that subepithelial vessels become visible after applying of green filter. 
The visibility of subepithelial vessels in white light is possible in cases of thinner atrophic epithelium [23] or in regeneration zones, where the epithelium has not yet reached its correct thickness [19]. Red colouration of both oral and cervical mucosae in inflammation, without coexisting precancerous lesions is the sign of correct transparency of these structures, because the red colour results from subepithelial vessels overloaded with blood, which are visible through the epithelium.

An important finding of this study was the presence of subclinical symptoms of alterations (punctation or mosaic subepithelial capillaries) detected by direct oral microscopy, which were not detected by a thorough clinical examination. In two-thirds of the patients presenting these alterations, the presence of dysplasia was confirmed histologically, mainly as low-grade dysplasia, nevertheless in one patient, moderate dysplasia was revealed. These data are in agreement with colposcopic observations of cervical mucosae, where punctation or mosaic capillaries constitute an indicator for histopathologic verification, as they may be a sign of high-grade dysplasia or even preinvasive cancer [1]. Our results suggest that improving direct oral microscopy as a method to support clinical examination of the oral cavity, may contribute to earlier detection and treatment of potentially malignant lesions of the oral mucosa.

\section{Conclusions}

We have obtained, by direct oral microscopy, a standard picture of healthy oral mucosae according to colposcopic criteria. The establishment of this standard picture of healthy oral mucosae constitutes a significant reference point for early subclinical detection of potentially malignant lesions. Application of in vivo tissue imaging techniques, commonly utilised in gynaecology, to investigate oral mucosae is a novel approach, which aims to increase the detection rate of oral precancerous lesions and oral cancers. This in turn may improve the treatment outcomes of such diseases. The availability of precise diagnostic equipment, together with growing social consciousness concerning oral cavity health, gives hope that these goals may be achieved in the near future.

\section{References}

1. Gynther GW, Rozell B, Heimdahl A. Direct oral microscopy and its value in diagnosing mucosal lesions. A pilot study. Oral Surg Oral Med Oral Pathol Oral Radiol Endod 2000; 90: 164-70.

2. Kmieć Z. Histologia zęba i jamy ustnej [Polish]. Publishing Skryptor, Gdansk 2003.

3. Tabor MP, Braakhuis BJM, van der Wal JE, et al. Comparative molecular and histological grading of epithelial dysplasia of the oral cavity and the oropharynx. J Pathol 2003; 199: 354-60.

4. Garzón I, Sánchez-Quevedo MC, Moreu G, et al. In vitro and in vivo cytokeratin patterns of expression in bioengineered human periodontal mucosa. J Periodont Res 2009; 44: 588-97.
5. L'Estrange P, Bevenius J, Williams L. Intraoral application of microcolpohysteroscopy. A new technique for clinical examination of oral tissues at high magnification. Oral Surg Oral Med Oral Pathol 1989; 67: 282-5.

6. Swinson B, Jerjes W, El-Maaytah M, et al. Optical techniques in diagnosis of head and neck malignancy. Oral Oncol 2006; 42: 221-8.

7. Gillenwater A, Papadimitrakopoulou V, Richards-Kortum R. Oral premalignancy: new methods of detection and treatment. Curr Oncol Rep 2006; 8: 146-54.

8. Grubisić G, Klarić P, Jokanović L, et al. Diagnostic approach for precancerous and early invasive cancerous lesions of the uterine cervix. Collegium Anthropologicum 2009; 33: 1431-6.

9. Tan JHJ, Wrede CDH. New technologies and advances in colposcopic assessment. Best Pract Res Cl Ob 2011; 25: 667-77.

10. Argenziano G, Ferrara G, Francione S, et al. Dermoscopy - the ultimate tool for melanoma diagnosis. Semin Cutan Med Surg 2009; 28: 142-8.

11. De Giorgi V, Massi D, Carli P. Dermoscopy in the management of pigmented lesions of the oral mucosa. Oral Oncol 2003; 39: 534-5.

12. Lin J, Koga H, Takata M, et al. Dermoscopy of pigmented lesions on mucocutaneous junction and mucous membrane. Br J Dermatol 2009; 161: 1255-61.

13. Mannone F, De Giorgi V, Cattaneo A, et al. Dermoscopic features of mucosal melanosis. Dermatol Surg 2004; 30: 1118-23.

14. Gencoglan G, Gerceker-Turk B, Kilinc-Karaarslan I, et al. Dermoscopic findings in Laugier-Hunziker syndrome. Arch Dermatol 2007; 143: 631-3.

15. Braun RP, Rabinovitz HS, Krischer J, et al. Dermoscopy of pigmented seborrheic keratosis: a morphological study. Arch Dermatol 2002; 138: 1556-60.

16. Florczak K. Rak szyjki macicy. Kolposkopia. Digital Medicine in the Future. A.D. 2009

17. Klimek R (ed.). Ginecology. PZWL, Warsaw 1982.

18. Luesley D, Downey G. Value of normal colposcopy after an abnormal cervical smear report. JLGTD 2009; 13: 33-7.

19. Phadnis SV, Atilade A, Bowring J, et al. Regeneration of cervix after excisional treatment for cervical intraepithelial neoplasia: a study of collagen distribution. BJOG Int J Obstet Gy 2011; 118: 1585-91.

20. O’Neill E, Reeves MF, Creinin MD. Baseline colposcopic findings in women entering studies on female vaginal products. Contraception 2008; 78: 162-6.

21. Burke L, Antonioli D. Vaginal adenosis. Factors influencing detection in a colposcopic evaluation. Obstet Gynecol 1976; 48: 413-21.

22. Górska R (ed.). Diagnostyka i leczenie chorób błony śluzowej jamy ustnej. Med Tour Press International, Otwock 2011.

23. Le Donne M, Caruso C, Mancuso A, et al. The effect of vaginally administered genistein in comparison with hyaluronic acid on atrophic epithelium in postmenopause. Arch Gynecol Obstet 2011; 283: 1319-23. 